\title{
Soluble inter-cellular adhesion molecule-1 in urban Asian north Indians: Relationships with anthropometric and metabolic covariates
}

\author{
Astha Sethi ${ }^{\mathrm{a}}$, Anoop Misra ${ }^{\mathrm{a}, *}$, Ravindra Mohan Pandey ${ }^{\mathrm{b}}$, Kalpana Luthra $^{\mathrm{c}}$, J. Rama Devi $^{\mathrm{a}}$, \\ Rekha Sharma $^{\mathrm{d}}$, Nidhi Khanna ${ }^{\mathrm{d}}$, Manjari Dwivedi ${ }^{\mathrm{c}}$ and Naval Kishore Vikram ${ }^{\mathrm{a}}$ \\ Departments of ${ }^{\mathrm{a}}$ Medicine, ${ }^{\mathrm{b}}$ Biostatistics, ${ }^{\mathrm{c}}$ Biochemistry and ${ }^{\mathrm{d}}$ Dietetics, All India Institute of Medical Sciences, \\ New Delhi-110 029, India
}

\begin{abstract}
Background: High prevalence of diabetes, obesity, and dyslipidemias in people belonging to poor socio-economic strata in urban slums of northern India has been recorded recently. To assess whether this population has high levels of soluble intercellular adhesion molecule-1 (sICAM-1), a cytokine involved in the pathogenesis of atherosclerosis, we investigated subjects belonging to poor socio-economic strata in urban slums and compared them to healthy control subjects from non-slum urban areas of New Delhi.

Design: Cross-sectional study.

Methods: Subjects from a previously carried out cross-sectional study, Delhi Urban Slum Project (DUSP) were divided into two groups: Group-1 $(n=56)$ included subjects dwelling in slum area, having at least one risk factor (hypertension, hyperglycemia, hypertriglyceridemia and hypercholesterolemia), while group-2 $(n=60)$ consisted of subjects without any risk factor dwelling in the slum area. A third group $(n=29)$ of non-obese subjects without any risk factor living in non-slum urban area was included for comparison. Measurements included; body mass index (BMI), waist-hip ratio (W-HR), four skinfolds, percentage body fat, fasting plasma glucose (FPG), serum lipids, and serum levels of sICAM-1.

Results: Though statistically not significant, mean level of sICAM-1 was higher in group-1 (718.5 $\pm 232.8 \mathrm{ng} / \mathrm{ml})$ as compared to the other groups. Of note, $35 \%$ of subjects in group- 1 ( $p<0.05$ as compared to other two groups), and $25.3 \%$ of all subjects had levels of sICAM-1 in uppermost quartile ( $>850 \mathrm{ng} / \mathrm{ml})$. Partial correlation coefficients (R) of sICAM-1 levels with various parameters adjusted for age were statistically significant for BMI $(R=0.27, p<0.05)$ in group-1; W-HR $(R=0.26, p<0.05)$ and BMI $(R=0.19, p<0.05)$ for group-2; and FPG $(R=0.17, p<0.05)$ for all the subjects considered together. For females, the levels of sICAM-1 were significantly higher in the following: BMI $\geqslant 25 \mathrm{~kg} / \mathrm{m}^{2}(p=0.04)$ and FPG $>7 \mathrm{mmol} / 1$ $(p<0.05)$. Multiple linear regression analysis suggests that an increment in BMI by one $\mathrm{kg} / \mathrm{m}^{2}$ would correspond to an increase in the levels of sICAM-1 by 8.5 units controlling for the influence of age and W-HR in the pooled data of all subjects.

Conclusions: High percentage of subjects had levels of sICAM-1 in the upper quartile in the study, particularly those dwelling in the slum area and having coronary risk factor (s). The levels of sICAM-1 strongly correlated to the anthropometric and metabolic parameters, particularly in females. These observations are of potential importance for the pathogenesis of atherosclerosis in this population, though further studies are needed to predict those prone to the complications of atherosclerosis, based on sICAM-1 levels, as has been observed in other ethnic groups.
\end{abstract}

Keywords: Soluble intercellular adhesion molecule-1, body mass index, obesity, type 2 diabetes mellitus, hyperlipidemia

* Address for correspondence: Anoop Misra, MD, Center for Human Nutrition, Department of Internal Medicine, The University of Texas Southwestern Medical Center at Dallas, 5323, Harry Hines Blvd., Dallas, TX 75390-9052, USA. Tel.: +1 214648 3198; Fax: +1 214648 7150; E-mail: anoopmisra@ @otmail.com.

\section{Introduction}

Recent evidence suggests that the inflammatory pathways are important for the pathogenesis of atherosclerosis. There is a growing body of evidence supporting the role of cell adhesion molecules (CAMs) in the 
initiation of atherosclerosis. CAMs are expressed on the endothelial membrane in response to inflammatory cytokines [17] and promote leucocyte adhesion to vascular endothelium and subsequent transmigration [12, 27], thus initiating the formation of 'foam cells'. Immunohistochemical assays in the hyperlipidemic experimental models show increased expression of adhesion molecules in atherosclerotic plaques [25]. Recent studies using chromatin image analysis provide further important evidence regarding sICAM and inflammatory nature of atherosclerosis [9]

High serum levels of CAMs may indicate endothelial dysfunction [14], an important feature of insulin resistance syndrome (IRS) and associated metabolic diseases [7]. Recent studies show upregulation of CAMs in obese hypertensive men [10] and in the patients with type 2 diabetes mellitus and coronary artery disease (CAD) [29]. Among various types of CAMs, soluble intercellular cell adhesion molecule-1 (sICAM1) appears to be more specific to the atherosclerotic vascular lesions [5]. High serum levels of sICAM-1 have been reported in communicable and other noncommunicable diseases, e.g. bacterial and viral infections $[1,15]$ and respiratory disorders [23].

Asian Indians are particularly vulnerable to develop IRS and accelerated atherosclerosis. High prevalences of obesity, type 2 diabetes mellitus, and dyslipidemia have been reported in urban subjects and also in economically poor people residing in urban slums [4]. Such people undergo marked socio-economic transition, having migrated from villages to cities in the search of better livelihood. During this process they relinquish their simple penurious and physically active lifestyle, and acquire several risk factors of atherosclerosis e.g. adverse dietary profile, sedentary habits, smoking, and alcohol intake. In addition, they undergo severe psychological stress. Recently, high prevalence of risk factors for atherosclerosis and pro-atherogenic dietary profile of the urban slum population have been reported by us $[3,4]$. Similarly, studies in other ethnic groups also sugget that obesity and malnutrition co-exist in urban slums [8].

We studied two groups of subjects, with and without coronary risk factors (type 2 diabetes mellitus, hypertension, and dyslipidemia) from the previously carried out Delhi Urban Slum Project (DUSP), and compared them to a control group consisting of healthy subjects from urban non-slum area of New Delhi. We also examined relationships of serum levels of sICAM-1, anthropometric and metabolic parameters.

\section{Methods}

Three groups of subjects $(n=145)$ from two different residential areas in Southern New Delhi, based on the presence of any of the following risk factors for CAD namely hypertension, hyperglycemia, hypertriglyceridemia and hypercholesterolemia were included in the study. Group-1 $(n=56)$ consisted of subjects from urban slum area having at least one of the previously mentioned risk factors. Group-2 $(n=60)$ comprised of subjects from urban slum area having none of the risk factors mentioned above. The sanitary and hygienic conditions in the slum area were extremely poor, and the population frequently suffered from common infectious diseases (respiratory and gastrointestinal infections and enteric fever etc.) and malaria. Subjects in group-3 ( $n=29$ ) were non-obese, non-diabetic medical students living in urban non-slum area, having none of the risk factors and had no history any acute infections (within previous six months) or chronic infections (respiratory, gastrointestinal and others).

Group-1 and group-2 subjects were selected from the sample of population recruited for Delhi Urban Slum Project (DUSP) [4]. Out of 532 subjects initially recruited in DUSP, 116 subjects were included in groups 1 and 2 in the present study based on their risk factor profile and adequacy of frozen serum sample for analysis of sICAM-1 levels. The slum area, (Gautam Nagar colony), consisted of sixteen square kilometer area, having a population of approximately 30,000 people. People had migrated from the adjoining states, and were residing in the slum colony for a mean period of 14 months (range 45 days-5 years). Their monthly incomes in rupees were as follows; no earning $-25 \%,<$ 1000 (approximately US \$25) -13\%, 1000-2000 (approximately US \$20-40) -32\%, 2000-4000 (approximately US \$40-80) - 20\%, > 4000 (approximately US \$85) $-10 \%$.

The detailed epidemiological procedures have been described elsewhere [4]. Briefly, the area was divided into four equal sectors using electoral list providing the name, age and addresses of those eligible for voting ( $>18$ years of age) available from the Slum Development Wing, Government of India. The data were collected within a period of 20 months, starting from January 1998. Two medical teams administered detailed questionnaires incorporating demographic profile, socio-economic data, migration pattern, relevant symptoms, tobacco and alcohol consumption, physical activity pattern, and food frequency profile to the recruited subjects. Subjects in group-3 were recruited 
by advertisements in the medical school, whereupon, healthy medical students were selected for the study. Subjects with manifest CAD, history of cerebrovascular accident, peripheral vascular disease, severe endorgan dysfunction, acute illnesses, acquired immunodeficiency syndrome, pregnant females, and patients of substance abuse etc. were excluded. The institutional ethics review board approved the study. An informed consent in the local language was taken from all the subjects.

Complete physical examination was performed in all the subjects. The subjects from the slum area were examined at the site, and medical students were admitted for a short period in the General Clinical Research Center. Blood pressure was recorded in sitting position after 5 minutes rest with a mercury sphygmomanometer according to the standard guidelines. If one blood pressure reading $\geqslant 140 / 90 \mathrm{mmHg}$ was observed, a second reading was taken after 10 minutes of rest. The blood samples were collected after 12-hour overnight fast for the estimation of the metabolic parameters.

\subsection{Anthropometric measurements}

Weight and height were measured to the nearest kilograms and to the nearest centimeter, respectively. The body mass index (BMI) was calculated as weight $(\mathrm{kg}) /$ height $^{2}(\mathrm{~m})$. Waist circumference was measured midway between iliac crest and lowermost margin of the ribs. The hip circumference was measured with the subject wearing minimum clothes possible at the maximum circumference of buttocks. Mean of three readings of each parameter was taken for the calculation of waist-hip ratio (W-HR). Lange skinfold calipers (Beta Technology Inc, Santa Cruz, CA, USA) were used to measure all skinfolds (biceps, triceps, subscapular, and suprailiac). With right arm pendant, fat pad was measured at the level of the nipple line for biceps skinfold. Triceps skinfold was measured midway between acromion process of scapula and olecranon process. Subcutaneous fat pads at the inferior angle of scapula and superiorly on iliac crest directly in the mid-axillary line were measured for sub-scapular and supra-iliac skinfolds, respectively. The skinfolds were measured to the nearest $1-\mathrm{mm}$ and a mean of three readings at each site was recorded. By adding all skinfolds, sum of four skinfolds (sigma 4 SF) was calculated. Percentage body fat (\% BF) was calculated using the equation of Durnin and Womersely [19]. For the calculation of body fat, the equation been validated in Asian Indians [31].

\subsection{Biochemical tests}

Blood samples were collected and transported to laboratory within 30 minutes. The samples were allowed to clot at room temperature for 45 minutes and serum was separated by centrifuging the tubes at $2000 \mathrm{rpm}$. for 15 minutes. Serum was stored frozen at $-20^{\circ} \mathrm{C}$ until further use. Estimations for total cholesterol (TC), serum triglycerides (TG), and high-density lipoprotein cholesterol (HDL-c) were performed on the sample drawn after a 12-hour overnight fast. TC, TG, and HDL-c were estimated by the ELISA kits (Randox Laboratory, San Francisco, CA, USA) on a semiautomated analyzer (Micro Semi-Autoanalyser 2000, C.L. Micromed, Italy). Value of low-density lipoprotein cholesterol (LDL-c) was calculated using Friedewald's equation.

\section{3. sICAM-1 assay}

The laboratory personnel performing the assay were masked of the group identity of the subjects. Stored sera from the subjects were thawed and assayed for sICAM-1 using ELISA kit (Batch 0054-23, Diaclone Research, France). The color developed after the reaction was read using an ELISA Reader (Microspan, India.) A linear curve was obtained by plotting the average absorbance, depicted by optical density (OD) or the vertical axis versus the corresponding sICAM-1 standard concentration on horizontal axis. The amount of sICAM-1 in each sample was determined by extrapolating OD values to sICAM-1 concentrations using the standard curve. The minimal detectable dose of sICAM-1 according to the manufacturer was $0.1 \mathrm{ng} / \mathrm{ml}$. The average normal level of sICAM as given by the manufacturers was $571 \pm 168 \mathrm{ng} / \mathrm{ml}$ (range: 219 to $1042 \mathrm{ng} / \mathrm{ml})$.

\subsection{Definitions}

Obesity was defined as BMI $>25 \mathrm{~kg} / \mathrm{m}^{2}$ [42]. \% $\mathrm{BF}$ was defined as indicative of obesity if it was $>$ $25 \%$ in males and $>30 \%$ in females $[26,38]$. High $\mathrm{W}-\mathrm{HR}$ was defined as $>0.95$ in males and $>0.80$ in females [41]. Sigma 4SF was defined as high when the value exceeded $50 \mathrm{~mm}$ [40]. A subject was labeled as hypertensive when systolic blood pressure was $\geqslant 140 \mathrm{~mm} \mathrm{Hg}$, and diastolic blood pressure was $\geqslant$ $90 \mathrm{~mm} \mathrm{Hg}$ on two measurements taken ten minutes apart in previously undiagnosed patients, and those patients already on anti-hypertensive treatment. Dia- 
betes mellitus was defined as fasting plasma glucose $(\mathrm{FPG}) \geqslant 7.0 \mathrm{mmol} / \mathrm{l}(126 \mathrm{mg} / \mathrm{dl})$ [39]. Hyperlipidemia was defined by the following; serum triglycerides (TG) $\geqslant 2.26 \mathrm{mmol} / \mathrm{l}(200 \mathrm{mg} / \mathrm{dl})$, total cholesterol $(\mathrm{TC}) \geqslant$ $5.20 \mathrm{mmol} / \mathrm{l}(200 \mathrm{mg} / \mathrm{dl})$, or LDL-c $\geqslant 3.88 \mathrm{mmol} / \mathrm{l}$ $(150 \mathrm{mg} / \mathrm{dl})$ [28].

\subsection{Statistical analysis}

Data were recorded on a pre-designed proforma and managed on Excel spreadsheet. All the entries were double-checked for any possible error by the same person. STATA 7.0 Intercooled version (Stata Corporation, College Station, Houston, TX, USA) was used for the statistical analysis. Anthropometric variables, metabolic profile, and the values of sICAM-1 were considered at quantitative level for purpose of comparison amongst the three groups. The distribution of all the quantitative variables was assessed for approximate normality and after confirmation these were summarized by mean and standard deviation for each group. One-way analysis of variance (ANOVA) was used to compute anthropometric and metabolic parameters amongst the three groups. If the overall significance was shown by the ' $F$ ' test, Scheffe's multiple comparison was used as the post-hoc ANOVA to assess which pair(s) of mean values was different. Partial correlation coefficient was computed to assess the strength of relationship between SICAM-1 and various anthropometric and metabolic parameters adjusted for age. Age adjustment was carried out as the subjects in three groups were of different age. The mean values of sICAM-1 for various subgroups in the present study were compared with similar subgroups in other studies by modified student's ' $\mathrm{t}$ ' test with $n 1+n 2-2$ degrees of freedom (used in case of unequal variance) [30]. Stepwise multiple linear regression analysis was used to determine the predictors for the levels of sICAM1. Value of $p<0.05$ was considered as statistically significant.

\section{Results}

\subsection{Demographic and anthropometric data}

Mean age (years) of subjects in group-1 $(42.3 \pm 11.1)$ was significantly higher $(p<0.001)$ as compared to the subjects in group-2 (36.9 \pm 13.1$)$ and group-3 (23.0 $\pm 4.0)$. The gender distribution of subjects in the three groups was statistically comparable. The proportion of smokers was $23(41.1 \%), 23(38.3 \%)$ and $2(6.9 \%)$ in groups 1, 2 and 3, respectively (Table 1).

Obesity-related variables were analysed in group 1 and group- 2 only, since group-3 consisted of non-obese subjects. Group-1 subjects had significantly higher values of BMI as compared to other two groups $(p<0.01)$. Applying BMI $\geqslant 25 \mathrm{~kg} / \mathrm{m}^{2}$ as cutoff for the diagnosis of obesity, $4(7 \%)$ males and $15(25 \%)$ females were obese (excluding group-3). The group-wise distribution of obese subjects was as follows: group-1-males: $3(11.4 \%)$, females $10(33.3 \%)$, and group-2-males: $1(3.3 \%)$, females: $5(16.7 \%)$. Biceps skinfolds and triceps skinfolds were significantly higher in group-3 $(p<0.05)$ as compared to other groups. Values of sigma $4 \mathrm{SF}$ in the three groups were statistically comparable. Similarly, no significant differences were observed in the values of $\% \mathrm{BF}$ and $\mathrm{W}-\mathrm{HR}$ in the three groups. Higher values of W-HR were recorded in 9 $(13.6 \%)$ males and $33(44 \%)$ females when all the subjects were pooled together. Similarly, 36 (48\%) females and $8(12.3 \%)$ males showed higher values of $\%$ BF.

\subsection{Metabolic profile}

The presence of hypertension, diabetes mellitus, hypercholesterolemia and hypertriglyceridemia was observed in $22(39.3 \%), 7(12.5 \%), 38(67.8 \%)$ and 26 $(46.4 \%)$ of subjects, respectively, in group-1 (Table 1). Significantly higher mean values of FPG $(p<0.05)$, TC $(p<0.001)$, TG $(p<0.001)$, LDL-c $(p<0.001)$, and ratio of LDL-c/HDL-c $(p<0.001)$ were observed in group-1 as compared to other groups. Mean value of HDL-c was lower in group-1 as compared to the other groups $(p<0.05)$. HDL-c values of $<0.9 \mathrm{mmol} / \mathrm{l}$ $(35 \mathrm{mg} / \mathrm{dl})$ were recorded in $14(25 \%)$ subjects in group1 , and $9(15.0 \%)$ subjects in group- 2 .

\subsection{Values of SICAM-1 and its correlations with anthropometric and metabolic parameters}

Mean levels of sICAM-1, though higher in group-1 (718.5 $\pm 232.8 \mathrm{ng} / \mathrm{ml})$, were statistically comparable amongst the three groups (Table 1, Fig. 1). Further, the levels of sICAM-1 were higher in the subjects with hypercholesterolemia $(720.7 \pm 273.3 \mathrm{ng} / \mathrm{ml})$, hypertriglyceridemia $(759.2 \pm 232.3 \mathrm{ng} / \mathrm{ml})$, diabetes mellitus $(878.6 \pm 159.5 \mathrm{ng} / \mathrm{ml})$ and hypertension $(788.6 \pm$ $274.3 \mathrm{ng} / \mathrm{ml}$ ) in group- 1 as compared to the mean levels in the other groups (Table 1), though the differences were statistically not significant. Further, mean serum 
Table 1

Anthropometric and biochemical profile adjusted for age and BMI (all values in mean \pm SD)

\begin{tabular}{lcccc}
\hline Variables & $\begin{array}{c}\text { Group-1 } \\
(n=56 ; \mathrm{M}-26, \mathrm{~F}-30)\end{array}$ & $\begin{array}{c}\text { Group-2 } \\
(n=60 ; \mathrm{M}-30, \mathrm{~F}-30)\end{array}$ & $\begin{array}{c}\text { Group-3 } \\
(n=29 ; \mathrm{M}-13, \mathrm{~F}-16)\end{array}$ & $\mathrm{p}$ value \\
\hline Anthropometry & & & & \\
Body mass index $\left(\mathrm{kg} / \mathrm{m}^{2}\right)$ & $22.1 \pm 4.5$ & $19.6 \pm 4.5^{\mathrm{a}}$ & $20.7 \pm 4.6$ & $<0.01$ \\
Skinfolds (mm) Biceps & $6.7 \pm 4.9$ & $8.0 \pm 4.9$ & $9.3 \pm 5.0^{\mathrm{b}}$ & $\mathrm{NS}$ \\
$\quad$ Triceps & $13.0 \pm 6.4$ & $12.9 \pm 6.3$ & $16.0 \pm 6.4^{\mathrm{b}, \mathrm{c}}$ & $<0.05$ \\
$\quad$ Subscapular & $15.9 \pm 8.1$ & $17.0 \pm 8.0$ & $18.4 \pm 8.1$ & $\mathrm{NS}$ \\
$\quad$ Suprailiac & $18.3 \pm 9.1$ & $19.6 \pm 9.1$ & $17.1 \pm 9.2$ & $\mathrm{NS}$ \\
$\quad$ Sigma 4SF & $53.4 \pm 25.4$ & $56.9 \pm 25.4$ & $60.5 \pm 25.7$ & $\mathrm{NS}$ \\
Percentage body fat (\%) & $23.7 \pm 7.5$ & $24.0 \pm 7.4$ & $25.3 \pm 7.6$ & $\mathrm{NS}$ \\
Waist-hip ratio & $0.84 \pm 0.06$ & $0.83 \pm 0.06$ & $0.83 \pm 0.07$ & $\mathrm{NS}$ \\
Biochemistry (mmol/l) & & & $4.65 \pm 1.76^{\mathrm{b}}$ & $<0.05$ \\
Fasting plasma glucose & $5.55 \pm 1.74$ & $4.79 \pm 1.73^{\mathrm{a}}$ & $4.16 \pm 0.94^{\mathrm{b}}$ & $<0.001$ \\
Total cholesterol & $5.73 \pm 0.93$ & $4.15 \pm 0.93^{\mathrm{a}}$ & $1.19 \pm 0.83^{\mathrm{b}}$ & $<0.001$ \\
Serum triglycerides & $2.14 \pm 0.82$ & $1.28 \pm 0.81^{\mathrm{a}}$ & $2.76 \pm 1.10^{\mathrm{b}}$ & $<0.001$ \\
LDL-c & $3.94 \pm 1.09$ & $2.62 \pm 1.09^{\mathrm{a}}$ & $1.01 \pm 0.13^{\mathrm{b}}$ & $<0.05$ \\
HDL-c & $0.96 \pm 0.13$ & $1.02 \pm 0.12^{\mathrm{a}}$ & $2.7 \pm 1.3^{\mathrm{b}}$ & $<0.001$ \\
Ratio of LDL-c/HDL-c & $4.2 \pm 1.3$ & $2.6 \pm 1.2^{\mathrm{a}}$ & $688.7 \pm 235.8$ & $\mathrm{NS}$ \\
sICAM-1 (ng/ml) & $718.5 \pm 232.8$ & $696.2 \pm 232.3$ &
\end{tabular}

a: I Vs II; ${ }^{\text {b }}$ : I Vs III; ${ }^{c}$ : II Vs III; statistically different at $p<0.05$; NS: Statistically not significant. Group-1: Subjects from urban slums having at least one risk factor of coronary artery disease. Group-2: Non-diabetic, normolipidemic and normotensive subjects from urban slums. Group-3: Non-diabetic, non-obese, normolipidemic and normotensive controls from urban non-slum area. Sigma 4SF: sum of four skinfolds; sICAM-1: soluble intercellular adhesion molecule-1. LDL-c: low-density lipoprotein cholesterol; HDL-c: high-density lipoprotein cholesterol.

levels of sICAM-1 between the smokers $(706.3 \pm$ $251.7 \mathrm{ng} / \mathrm{ml})$ and non-smokers $(704.7 \pm 223.7 \mathrm{ng} / \mathrm{ml})$ were statistically not significant in all subjects combined and in each group. Subjects were categorized into quartiles of sICAM-1 levels (ng/ml) as quartile 1, $<$ 500; quartile 2, 501-730; quartile 3, > 731-850; and quartile $4,>851$ (Fig. 2). Number of subjects in the uppermost quartile was: group-1, $20(35.1 \%, p<0.05$ as compared to other two groups), group-2, 12 (20\%) and group-3, 5 (17.3\%). Of note, overall, 37(25.3\%) of subjects had sICAM-1 levels in uppermost quartile (Fig. 2).

Partial correlation coefficient $R$ between levels of sICAM-1 and various anthropometric and metabolic parameters adjusted for age showed significant $(p<$ $0.05)$ correlations with BMI in group-1 $(R=0.27)$, with W-HR in group-2 $(R=0.26)$, and with BMI ( $R=0.19)$ and FPG $(R=0.17)$ when subjects in three groups were pooled together.

Multiple linear regression analyses with serum sICAM-1 as the dependent variable were used to develop regression equations of all subjects (Table 2). Linear relationship between SICAM-1 levels and BMI was observed when all subjects were analyzed together. To assess the lipid parameters being influenced by the levels of sICAM-1, while keeping the effect of other variables on lipid parameters as constant, we used multiple linear regression analysis with individual lipid pa- rameters as dependent variables, sICAM-1 as an exposure variable and age, BMI, W-HR, area of residence, and other lipid parameters as co-variates. There was no statistically significant relationship of sICAM-1 with any of the lipid parameters. Subsequently, considering sICAM-1 as the response variable, we assessed the influence of anthropometric variables on sICAM-1 levels. Amongst age, BMI, waist-hip ratio, and area of residence, BMI was observed to be the only independent predictor of levels of sICAM-1. Increment in BMI value by one $\mathrm{kg} / \mathrm{m}^{2}$ would correspond to an increase in levels of sICAM-1 by 8.5 units $(p<0.05)$ controlling for age and W-HR.

For females, the levels of sICAM-1 were significantly higher $(831.3 \pm 247.5 \mathrm{ng} / \mathrm{ml})$ in those with BMI $\geqslant 25 \mathrm{~kg} / \mathrm{m}^{2}$ as compared to the subjects with BMI $<$ $25 \mathrm{~kg} / \mathrm{m}^{2}(p<0.05)$, and in those with high values of FPG $(906.6 \pm 154.6 \mathrm{ng} / \mathrm{ml})$ as compared to those with normal blood glucose levels $(p<0.05)$. No significant relationship of sICAM-1 levels and various anthropometric and metabolic parameters was observed in males.

\section{Discussion}

The observations of similar levels of sICAM-1 in people of low socio-economic strata with and without 


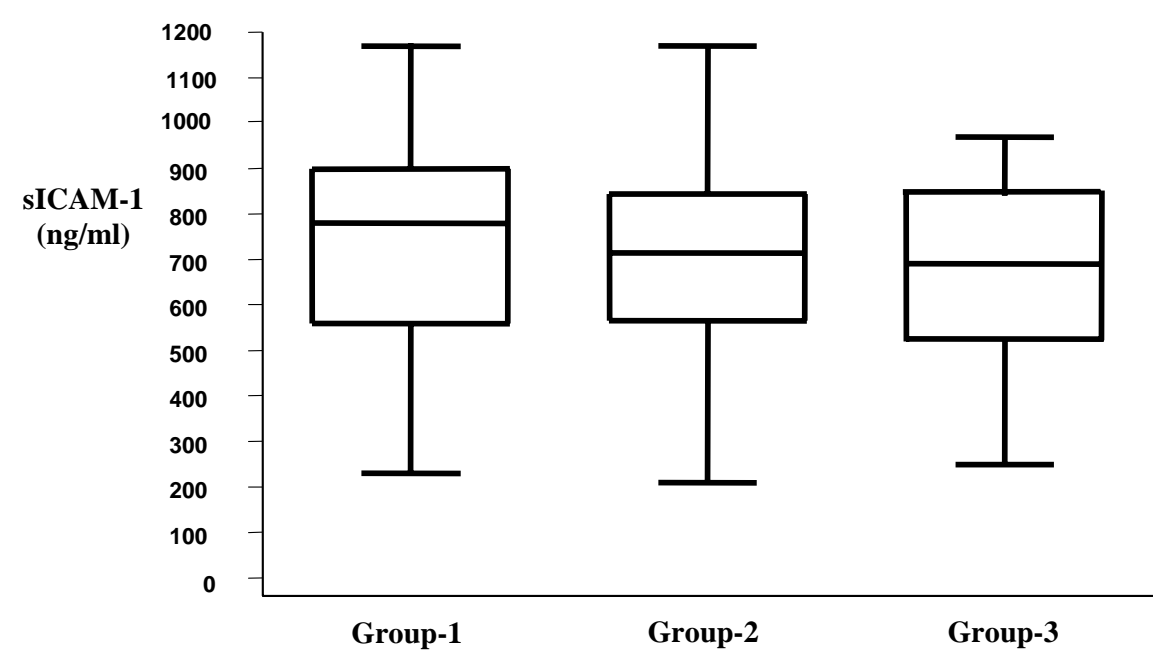

Fig. 1. Summary statistics of serum levels of sICAM-1. Group-1: Subjects from urban slums having at least one risk factor for coronary artery disease; group-2: Non-diabetic, normolipidemic and normotensive subjects from urban slums; group-3: Non-diabetic, normolipidemic and normotensive subjects from urban non-slum area; sICAM-1: Soluble intercellular adhesion molecule-1.

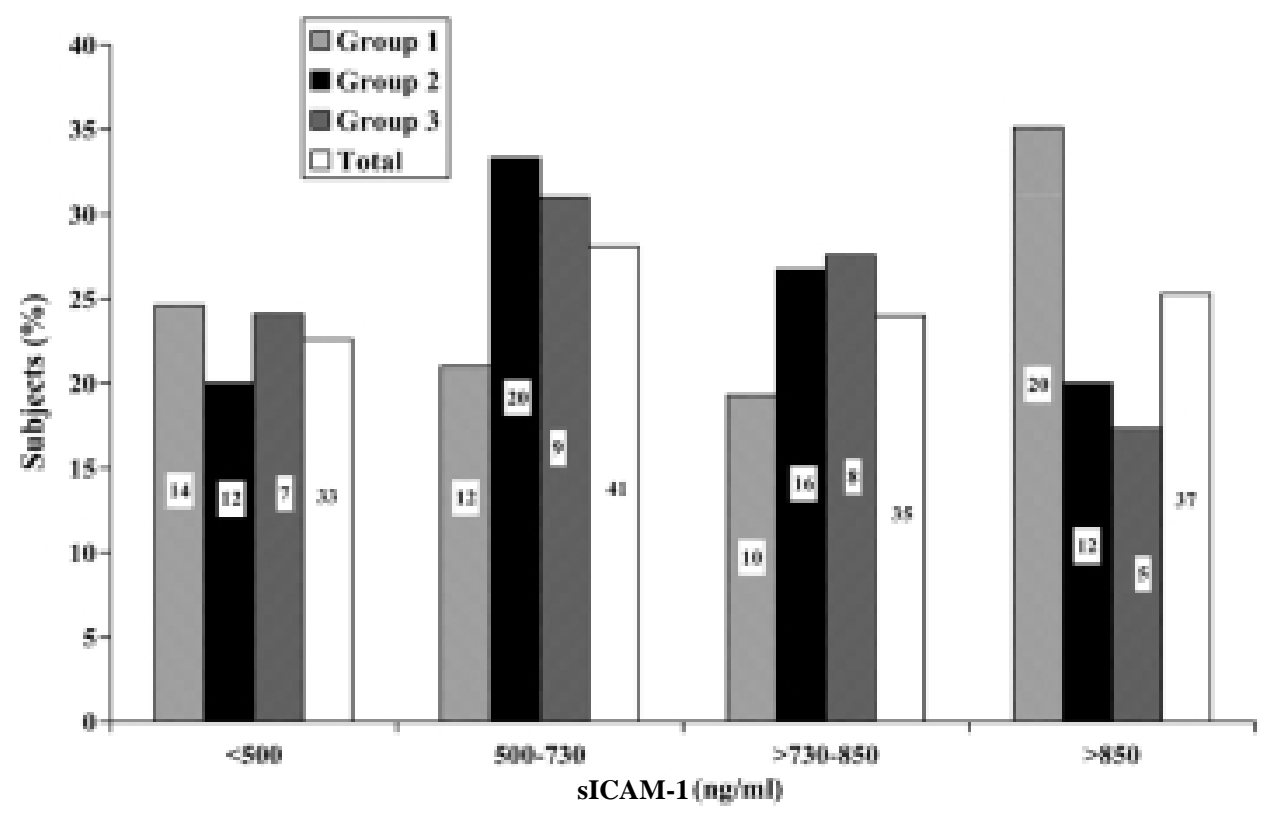

Fig. 2. Distribution of subjects according to quartiles of sICAM-1 levels. Group-1: Subjects from urban slums having at least one risk factor for coronary artery disease; group-2: Non-diabetic, normolipidemic and normotensive subjects from urban slums; group-3: Non-diabetic, normolipidemic and normotensive subjects from urban non-slum area; sICAM-1: Soluble intercellular adhesion molecule-1.

coronary risk factors as well as in normal subjects are of significance. Of note, in this study approximately $20-35 \%$ subjects displayed levels of sICAM-1in the highest quartile in all the groups, including the group consisting of young, health-conscious, normotensive, non-obese, non-diabetic medical students. Moreover, the low socio-economic strata group having various risk factors showed the highest number of subjects in the uppermost quartile of sICAM-1. These observations are of potential importance, since, according to the data in the Physicians Health Study, increasing concentrations of s1CAM-1 predict future risk for myocardial infarction (MI). According to Ridker et al. subjects in the highest quartile of sICAM-1 levels (taken as 75th 
Table 2

Multiple linear regression analysis with serum levels of sICAM-1 as the dependent variable

\begin{tabular}{|c|c|}
\hline Regression equation & $R^{2}(\%)$ \\
\hline $\mathrm{TC}=+38.5+0.009($ sICAM-1 $)+6.4($ SlumRes $)-0.03($ Age $)+0.05(\mathrm{BMI})+0.14^{*}(\mathrm{TG})+0.76^{*}(\mathrm{LDL}-\mathrm{c})$ & 73.3 \\
\hline $\mathrm{TG}=-71.5+0.01(\mathrm{sICAM}-1)+20.2($ SlumRes $)+0.83($ Age $)+167.9^{*}(\mathrm{~W}-\mathrm{HR})+1.6 *(\% \mathrm{BF})+0.88^{*}(\mathrm{~T} . \mathrm{C})-3.6^{*}(\mathrm{HDL}-\mathrm{c})$ & 33.1 \\
\hline HDL-c $=36.3+0.001($ sICAM-1 $)+0.39($ SlumRes $)-0.009($ Age $)+0.04(\mathrm{BMI})-0.02 *(\mathrm{TC})-0.01 *(\mathrm{TG})$ & 10 \\
\hline LDL-c $=-35.3-0.005\left(\right.$ sICAM-1) $-1.07($ SlumRes $)+0.24($ Age $)+0.71(\mathrm{BMI})+0.88(\mathrm{TC})^{*}-0.15^{*}(\mathrm{TG})$ & 71.7 \\
\hline sICAM-1 $=185.0+0.94($ Age $)+8.5 *(B M I)+342.0($ W-HR $)+5.2($ SlumRes $)$ & 5.3 \\
\hline
\end{tabular}

sICAM-1: Soluble intercellular adhesion molecule-1; BMI: body mass index; W-HR: Waist-hip ratio; TC: total cholesterol; TG: serum triglycerides; HDL-c: high-density lipoprotein cholesterol; LDL-c: low-density lipoprotein cholesterol; SlumRes: Area of residence (slum/ non-slum);

*: $p<0.05 ; R^{2}$ : coefficient of determination.

percentile of the control distribution of SICAM-1), had a relative risk of 1.6 for developing MI (95\% confidence interval $1.1-2.4 ; p=0.02$ ) [29]. The crude matched-pair analysis revealed that the risk of future MI was $60 \%$ higher in subjects having sICAM-1 levels in the highest quartile. The risk increased with the increasing length of follow up, suggesting involvement of sICAM-1 in the initial pathway of atherosclerosis. High levels of s1CAM-1 also correlated to high levels of pro-coagulant factors like serum fibrinogen, tissue type plasminogen activator antigen, and plasma homocysteine, augmenting the atherogenic risk several folds [29]. Further, in Atherosclerosis Risk in Communities (ARIC) Study, those with baseline sICAM-1 levels in the highest quartile had five times more risk of CAD as compared to the subjects having the lowest levels (relative risk 5.5, 95\% confidence intervals 2.5-12.2) [37].

Levels of sICAM-1 in the slum population and healthy individuals in the present study could be affected due to multiple factors. Besides obesity and hyperlipidemia, myriad infections, as prevalent in northern India, may potentially increase sICAM-1 concentrations in blood and tissues. These include gastrointestinal infection with Helicobacter pylori, malaria, tuberculosis etc. All of these, and in particular, H. pylori, are common in Indian subcontinent. Prevalence of $H$. pylori, estimated serologically by IgG and IgA antibodies, was approximately 80-90\% in the adult Asian Indian population [13]. Potable water and food supply in the hostel setting are frequently contaminated and are likely sources of asymptomatic infection in healthy resident medical students constituting group-3 in the present study. Of relevance, expression of sICAM-1 is increased in gastric epithelial cells in $80 \%$ of persons infected with $H$. pylori, decreasing significantly after its eradication [1], and constitutes predominant form of CAMs expressed [20]. Of importance, seropositivity of $H$. pylori has been reported to be associated with higher risk of CAD [21] and its eradication is reported to be beneficial [21,22]. Interestingly, H. pylori DNA was demonstrated in the atherosclerotic plaques in the carotid arteries. Moreover, sICAM-1 was expressed in $75 \%$ of such plaques, significantly higher as compared to $H$. pylori negative plaques [36]. Despite these evidences, relationship between $H$. pylori and atherosclerosis needs further support, however, it remains potentially important in context of the current study. In individuals exposed to multiple infectious agents, such as in the current study, 'aggregate pathogen load' (pathogen burden due to exposure to multiple infectious agents) is of further interest, since it is reported to correlate to endothelial dysfunction, atherosclerosis, and mortality [35].

None of these issues have been investigated previously in people of poor socio-economic strata in the slums. In a preliminary study in urban population of city of Pune in western India, levels of pro-inflammatory cytokines, interleukin- 6 and tumor necrosis factor- $\alpha$ were reported to be the highest in the urban slum dwellers [18]. Frequent respiratory and gastrointestinal infections were cited as the possible causes of increased cytokine expression and endothelial dysfunction. The authors remarked that the role of increased levels of cytokines in the pathogenesis of type 2 diabetes mellitus and dyslipidemia in Asian Indian population remains to be defined [18].

Second, and perhaps an equally important cause of increased levels of sICAM-1 across all the three groups in the present study, may be exposure of the local population to an excessive atmospheric pollution. In New Delhi, one of the world's 10 most polluted cities, vehicular exhaust contributes mostly to the atmospheric pollution. Interestingly, short-term exposure to the particulate diesel exhaust in healthy human volunteers upregulates inflammatory mediators, including sICAM1 , in bronchial tissues, indicative of marked systemic and pulmonary inflammatory response [34]. Location of slums and residence of medical students recruited in the present study were close to one of the most pol- 
Table 3

Comparative data of levels of sICAM-1 in various ethnic groups and diseases

\begin{tabular}{|c|c|c|c|c|}
\hline Author & Ethnic group & Number & Disease & sICAM-1(ng/ml) \\
\hline \multirow[t]{3}{*}{ Hackman et al. (1996) [2] } & Caucasians (USA) & 14 & Hypercholesterolemia & $298 \pm 29$ \\
\hline & & 13 & Hypertriglyceridemia & $342 \pm 31$ \\
\hline & & 13 & Controls & $198.0 \pm 14$ \\
\hline \multirow[t]{2}{*}{ De Souza et al. (1997) [11] } & Caucasians (USA) & 11 & Hypertension & $232.4 \pm 16.5$ \\
\hline & & & Controls & $189.8 \pm 11.1$ \\
\hline \multirow[t]{3}{*}{ Ferri et al. (1999) [10] } & Europeans (Italy) & 16 & $\begin{array}{l}\text { Impaired glucose tolerance } \\
+ \text { +Hyperlipidemia }\end{array}$ & $268 \pm 84.1$ \\
\hline & & 25 & Impaired glucose tolerance & $268 \pm 84.1$ \\
\hline & & 28 & Hyperlipidemia & $195.1 \pm 68$ \\
\hline \multirow[t]{2}{*}{ Abe et al. (1998) [42] } & Caucasians (USA) & 39 & Hypertriglyceridemia and low HDL-C & $316.0 \pm 28.8$ \\
\hline & & & Controls & $225.0 \pm 16.6$ \\
\hline Kvasnicka (1998) [16] & Czechs & 118 & Type 2 diabetes mellitus & $307.71 \pm 86.2$ \\
\hline \multirow[t]{2}{*}{ Ridker et al. (1998) [29] ${ }^{\mathrm{a}}$} & Caucasians (USA) & 474 & Cases $^{\mathrm{b}}$ & $59-675^{\mathrm{d}}$ \\
\hline & & 474 & Controls $^{c}$ & $59-675^{\mathrm{d}}$ \\
\hline Kado et al. (1999) [32] & Asians (Japan) & 64 & Type 2 diabetes mellitus & $378.2 \pm 70$ \\
\hline Romano et al. (2000) [24] & Europeans (Italy) & 26 & Hypercholesterolemia & $264 \pm 75$ \\
\hline \multirow[t]{2}{*}{ John et al. (2000) [31] } & Europeans (Germany) & 52 & Hypercholesterolemia & $196 \pm 56$ \\
\hline & & 43 & Control group & $180 \pm 38$ \\
\hline \multirow[t]{9}{*}{ Present Studye (2001) } & Asians (Northern India) & 56 & Group- $1^{\mathrm{f}}$ & $705.0 \pm 261.4$ \\
\hline & & 60 & Group- $2^{f}$ & $680.5 \pm 226.9$ \\
\hline & & 29 & Group- $3^{\mathrm{f}}$ & $671.0 \pm 215.2$ \\
\hline & & & Subgroups & \\
\hline & & & 1. Hypertriglyceridemia and low & \\
\hline & & 9 & HDL-c & $691.8 \pm 263.8$ \\
\hline & & 40 & 2. Hypercholesterolemia & $712.0 \pm 250.0$ \\
\hline & & 27 & 3. Hypertriglyceridemia & $784.9 \pm 244.5$ \\
\hline & & 21 & 4. Hypertension & $806.8 \pm 236.6$ \\
\hline
\end{tabular}

${ }^{a}$ : Prospective study, all the subjects were divided in four quartiles. Risk of future MI was $80 \%$ higher for participants in highest quartile. b: Cases-Subjects who developed a first myocardial infarction after 9 years of follow-up. ${ }^{c}$ : Controls-Subjects who remained healthy throughout the 9-year follow-up. ${ }^{\mathrm{d}}$ : Range of sICAM-1 levels in case and control groups. ${ }^{\mathrm{e}}$ : Normal range of sICAM-1 level given by the manufacturer was $219-1042 \mathrm{ng} / \mathrm{ml}$. ${ }^{\mathrm{f}}$ : Group-1: Subjects from urban slums having $\geqslant 1$ risk factor for coronary artery disease; Group-2: Non-diabetic, normolipidemic and normotensive subjects from urban slums; Group-3: Non-diabetic, non-obese, normolipidemic and normotensive healthy controls from non-slum area. HDL-C: High-density lipoprotein cholesterol.

luted areas in the city. Upon long-term exposure to diesel exhaust as well as to other atmospheric pollutants, pulmonary and systemic levels of sICAM-1 may even be raised to a higher level. The latter is a potentially important yet unexplored problem for the people chronically living in the heavily polluted environment.

Table 3 shows inter-ethnic and inter-disease comparisons in the levels of sICAM-1 from the published literature $[2,11,16,24,32,33,43]$. It must be mentioned, however, that sICAM-1 levels were estimated in widely varied clinical situations and using different commercial kits and methodologies in the studies. For example, observation of higher values of SICAM-1 in the current study as compared to other studies could also be to the differences in the normal levels of SICAM-1 provided by the manufacturer of the ELISA kits used by us as compared to those provided by other manufacturers. Hence, data of sICAM-1 in sub-groups of patients in the present study, such as hypertension, diabetes mellitus and dyslipidemia could not be statistically com- pared to the matched groups from the studies carried out on the other ethnic groups.

The present study has its limitation because of its cross-sectional design and relatively limited number of subjects in each group. Further, temporal relationship between sICAM-1 levels and various factors influencing its expression and its relationship with endpoints of atherosclerosis were not estimated. A prospective study with larger number of subjects, including other inflammatory markers (e.g. C-reactive protein) and pro-coagulant markers (e.g. serum fibrinogen) of atherosclerosis is could yield more meaningful results. The sample population in the present study was also not matched for smoking status. Cigarette smoke is one of the most important factors causing increased sICAM-1 expression [6]. Though this may be one of the confounding factors, however, in the current study sICAM1 levels in smokers $(706.3 \pm 251.7 \mathrm{ng} / \mathrm{ml})$ were similar to those in non-smokers $(704.7 \pm 223.7 \mathrm{ng} / \mathrm{ml}, p=\mathrm{ns})$ in the pooled data of all subjects. Further, there was no 
difference when sICAM-1 levels were compared between smokers and non-smokers in each group. This anomalous observation in the study could be due to the small sample size. Further, uniform high exposure to atmospheric and indoor pollution to smokers as well as the non-smokers, and its potential effects on the levels of sICAM, could also be responsible for the leveling of the difference.

Significant correlations of serum levels of sICAM-1 with BMI and FPG in females were not surprising and consistent with the earlier studies on the same population sample showing higher coronary risk factors in females as compared to males $[3,4]$. Particularly striking observations were generalized and abdominal obesity and excess $\% \mathrm{BF}$, and steep increase in body fat in middle age in females [4]. Of further note, $12-28 \%$ of the females in this population were hyperlipidemic [4].

\section{Conclusions}

Significant observations of the current study were high number of subjects in the uppermost quartile of sICAM-1 levels in all groups, and particularly in group-1 and its significant correlation to parameters of generalized obesity, abdominal obesity, and glycemia, though mean levels of sICAM-1 were not significantly different among the three groups. Such high levels of sICAM-1 could also be due to the frequent infections and high levels of atmospheric pollution. In view of these observations, presence of high levels of sICAM1 may not be able to predict precisely those prone to atherosclerosis and its complications, as has been reported for other ethnic groups, although it may have a potential role in the pathogenesis of atherosclerosis in Asian Indians. This important research question should be addressed in large prospective studies.

\section{Acknowledgements}

The authors express their appreciation to Mrs. Jyoti for typing and editing the manuscript, and Mr. Ramesh Giri for supervising the study in slums and in the hospital. The authors also wish to thank the staff of SRB Centre of Clinical Pharmacology, Department of Medicine, All India Institute of Medical Sciences, New Delhi, including Mr. Inder Taneja, Mr. Gian Chand and Mrs. Alice Jacob for performing investigations, Mr. R.L Taneja for performing quality control of biochemical tests, and Mr. Surinder for the laboratory work. Help and volun- tary services provided from the volunteers, in particular the late Mr. Jaipal, Mr. Rohtash, and the population of the slum community is appreciated.

The study was partially supported by a financial grant from Science and Society Division, Department of Science and Technology, Ministry of Science and Technology, Government of India.

\section{References}

[1] A. Archimandritis, S. Sougioultzis and P.G. Foukas et al., Expression of HLA-DR, costimulatory molecules B7-1, B7-2, intercellular adhesion molecule-1 (ICAM-1) and Fas ligand (FasL) on gastric epithelial cells in helicobacter pylori gastritis, influence of H. pylori eradication, Clin Exp Immunol 119(3) (2000), 464-471.

[2] A. Hackman, Y. Abe and W. Insull et al., Levels of soluble cell adhesion molecules in patients with dyslipidemia, Circulation 93(7) (1996), 1334-1338.

[3] A. Misra, R. Sharma and R.M. Pandey et al., Adverse profile of dietary nutrients, anthropometry and lipids in urban slum dwellers of northern India, Eur J Clin Nutr 55(9) (2001), 727734.

[4] A. Misra, R.M. Pandey and J. Rama Devi et al., A high prevalence of diabetes, obesity, and dyslipidemia in urban slum population in northern India, Int J Obes 25 (2001), 1722-1729.

[5] A.C. van der Wal, P.K. Das, A.J. Tigges and A.E. Becker, Adhesion molecules on the endothelium and mononuclear cells in human atherosclerotic lesions, Am J Pathol 141(6) (1992), 1427-1433.

[6] A.D. Blann, C. Steele and C.N. McCollum, The influence of smoking on soluble adhesion molecules and endothelial cell markers, Thromb Res 85(5) (1997), 433-438.

[7] A.E. Hak, H.A. Pols, C.D. Stehouwer and J et al., Markers of inflammation and cellular adhesion molecules in relation to insulin resistance in nondiabetic elderly: the Rotterdam study, J Clin Endocrinol Metab 86(9) (2001), 4398-4405.

[8] A.L. Sawaya, G. Dallal and G. Solymos et al., Obesity and malnutrition in a shantytown population in the city of Sáo Paulo, Brazil, Obes Res 3(2) (1995), 135s-143s.

[9] A.I. Teplyakov, A.V. Pryshchepava and T.I. Chegerova, Chromatin image analysis provides new evidence of the relation of lymphocytes to cytokines and sCAM in the inflammatory nature of atherosclerosis, Ann N Y Acad Sci 947 (2001), 377381.

[10] C. Ferri, G. Desideri and M. Valenti et al., Early upregulation of endothelial adhesion molecules in obese hypertensive men, Hypertension 34 (1999), 568-573.

[11] C.A. DeSouza, D.R. Dengel and R.F. Macko et al., Elevated levels of circulating cell adhesion molecules in uncomplicated essential hypertension, Am J Hypertens 10(12 Pt 1) (1997), 1335-1341.

[12] D.H. Adams and S. Shaw, Leucocyte-endothelial interactions and regulation of leucocyte migration, Lancet 343 (1994), $831-835$.

[13] G. Kang, D.P. Rajan and S. Patra et al., Use of serology, the urease test, and histology in diagnosis of Helicobacter pylori infection in symptomatic and asymptomatic adults, Ind J Med Res 110 (1999), 86-90.

[14] G. Lupatteli, R. Lombardini and G. Schillaci et al., Flow mediated vasoactivity and circulating adhesion molecules in hyper- 
triglyceridemia: association with small dense LDL cholesterol particles, Am Heart J 140(3) (2000), 521-526.

[15] J. Bella and M.G. Rossmann, Review: rhinoviruses and their ICAM receptors, J Struct Biol 128(1) (1999), 69-74.

[16] J. Kvasnicka, J. Skrha and J. Perusicova et al., Haemostasis, cytoadhesive molecules (sE-selection and sICAM-1) and inflammatory markers in non-insulin dependent diabetes mellitus (NIDDM), Sb Lek 99(2) (1998), 97-101.

[17] J.S. Pober, M.A. Gimbrone and L.A. Lapierre et al., Overlapping patterns of activation of human endothelial cells by interleukin 1, tumor necrosis factor, and immune interferon, $J$ Immunol 137(6) (1986), 1893-1896.

[18] J.S. Yudkin, C.S. Yajnik and V. Mohamed-Ali et al., High levels of circulating proinflammatory cytokines and leptin in urban, but not rural, Indians, Diab Care 22(2) (1999), 363364.

[19] J.V.G.A. Durnin and J. Womersely, Body fat assessed from total body density and its estimation from skinfold thickness: measurements on 481 men and women aged from 16 to 72 years, Br J Nutr 32 (1972), 77-97.

[20] K. Higuchi, T. Arakawa and T. Uchida et al., In situ expression of cell adhesion molecules in chronic gastritis with $\mathrm{He}$ licobacter pylori infection, J Clin Gastroenterol 25(1) (1997), S215-S221.

[21] M. Kowalski, Helicobacter pylori (H. pylori) infection in coronary artery disease: influence of $\mathrm{H}$. pylori eradication on coronary artery lumen after percutaneous transluminal coronary angioplasty. The detection of $\mathrm{H}$. pylori specific DNA in human coronary atherosclerotic plaque, J Physiol Pharmacol 52(1) (2001), 3-31.

[22] M. Kowalski, P.C. Konturek and P. Pieniazek et al., Prevalence of Helicobacter pylori infection in coronary artery diseases and effect of its eradication on coronary lumen reduction after percutaneous coronary angioplasty, Dig Liver Dis 33(3) (2001), 222-229.

[23] M. Alvaro, Adhesion molecules and asthma, Allergol Immunopathol (Madr) 28(3) (2000), 110-115.

[24] M. Romano, A. Mezzetti and C. Marulli et al., Fluvastatin reduces soluble P-selectin and ICAM-1 levels in hypercholesterolemic patients: role of nitric oxide, J Invest Med 48(3) (2000), 183-189.

[25] M.I. Cybulsky and M.A. Gimbrone, Endothelial expression of mononuclear leukocyte adhesion molecule during atherogenesis, Science 251 (1990), 788-791.

[26] M.L. Pollock and J.H. Wilmore, Exercise in health and disease, WB Saunders, Philadelphia, 1990.

[27] M.P. Bevilacqua, R.M. Nelson and G. Mannori et al., Endothelial-leukocyte adhesion molecules in human disease, Annu Rev Med 45 (1994), 361-378.

[28] National Cholesterol Education Panel. Second Report of the Expert Panel on Detection, Evaluation, and Treatment of High Cholesterol in Adults. Adult Treatment Panel II, Circulation 89 (1994), 1329-1445.
[29] P.M. Ridker, C.H. Hennekens and B.R. Johnson et al., Plasma concentration of soluble intercellular adhesion molecule 1 and risks of future myocardial infection in apparently healthy men, Lancet 351 (1998), 88-92.

[30] P. Armitage, G. Berry and J.N.S. Mathews, Statistical Methods in Medical Research, (Fourth ed.), Blackwell Science Ltd., Oxford, 2002, pp. 109-111.

[31] R. Kuriyan, C. Petracchi and A. Ferro-Luzzi et al., Validation of expedient methods for measuring body composition in Indian adults, Ind J Med Res 107 (1998), 37-45.

[32] S. John, J. Jacobi and C. Delles et al., Plasma soluble adhesion molecules and endothelium-dependent vasodilation in early human atherosclerosis, Clin Sc 98 (2000), 521-529.

[33] S. Kado and N. Nagata, Circulating intercellular adhesion molecule-1, vascular cell adhesion molecule-1, and E-selectin in patients with type 2 diabetes mellitus, Diab Res Clin Pract 46(2) (1999), 143-148.

[34] S. Salvi, A. Blomberg and B. Rudell et al., Acute inflammatory responses in the airways and peripheral blood after short-term exposures to diesel exhaust in healthy human volunteers, Am J Respir Crit Care Med 159(3) (1999), 702-709.

[35] S.E. Epstein, The multiple mechanisms by which infection may contribute to atherosclerosis development and course, Circ Res 90 (2002), 2-4.

[36] S.F. Ameriso, E.A. Fridman, R.C. Leiguarda and G.E. Sevlever, Detection of Helicobacter pylori in human carotid atherosclerotic plaques, Stroke 32(2) (2001), 385-391.

[37] S.-J. Hwang, C.M. Ballantyne and A.R. Sharret et al., Circulating adhesion molecules VCAM-1, ICAM-1, and E-selectin in carotid atherosclerosis and incident coronary heart disease cases. The Atherosclerosis Risk in Communities (ARIC) Study, Circulation 96 (1997), 4219-4225.

[38] T. Hortobagyi, R.G. Israel and K.F. O'Brien, Sensitivity and specificity of the Quetelet index to assess obesity in men and women, Eur J Clin Nutr 48(5) (1994), 369-375.

[39] The Expert Committee on the Diagnosis and Classification of Diabetes Mellitus: Report of the Expert Committee on Diagnosis and Classification of Diabetes Mellitus, Diab Care 20(7) (1997), 1183-1197.

[40] V. Dudeja, A. Misra and R.M. Pandey et al., Body mass index does not accurately predict overweight in Asian Indians in Northern India, Bri J Nutr 86 (2001), 105-112.

[41] W.C. Willett, W.H. Dietz and G.A. Colditz, Guidelines for Healthy Weight, $N$ Engl J Med 341 (1999), 427-434.

[42] World Health Organization, The Asia-Pacific Perspective. Redefining obesity and its treatment. International Diabetes Institute. Health Communications Australia Pty Limited, February 2000 .

[43] Y. Abe, B.E. Masri and K.T. Kimball et al., Soluble cell adhesion molecules in hypertriglyceridemia and potential significance on monocyte adhesion, Arterioscler Thromb Vasc Biol 18 (1998), 723-731. 


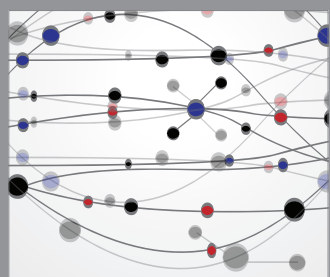

The Scientific World Journal
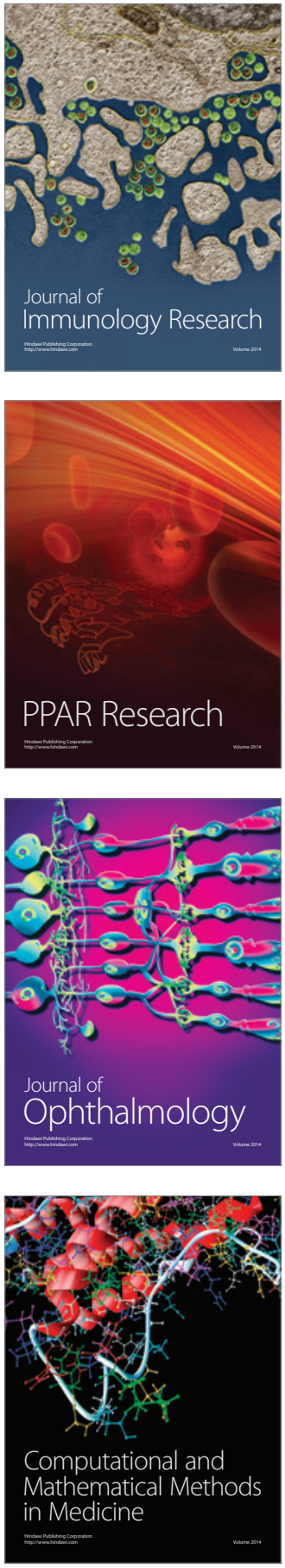

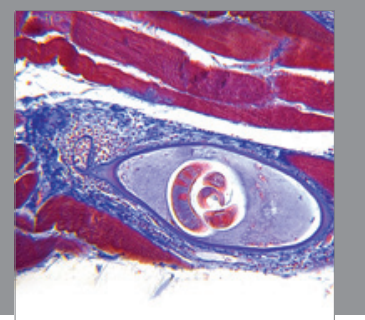

Gastroenterology

Research and Practice
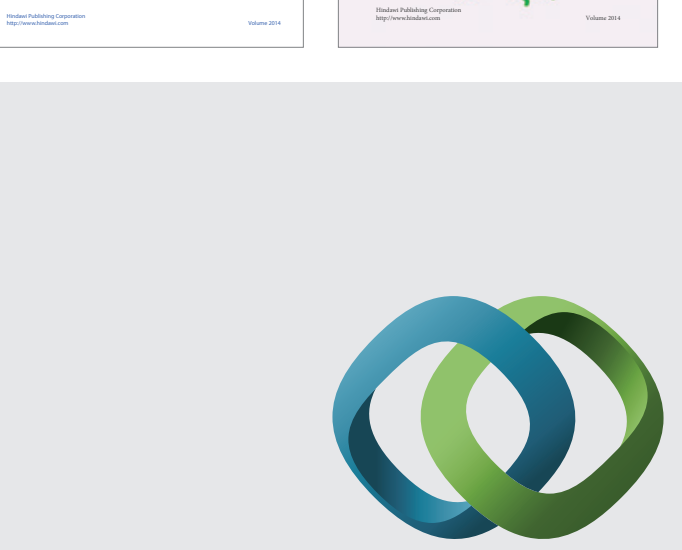

\section{Hindawi}

Submit your manuscripts at

http://www.hindawi.com
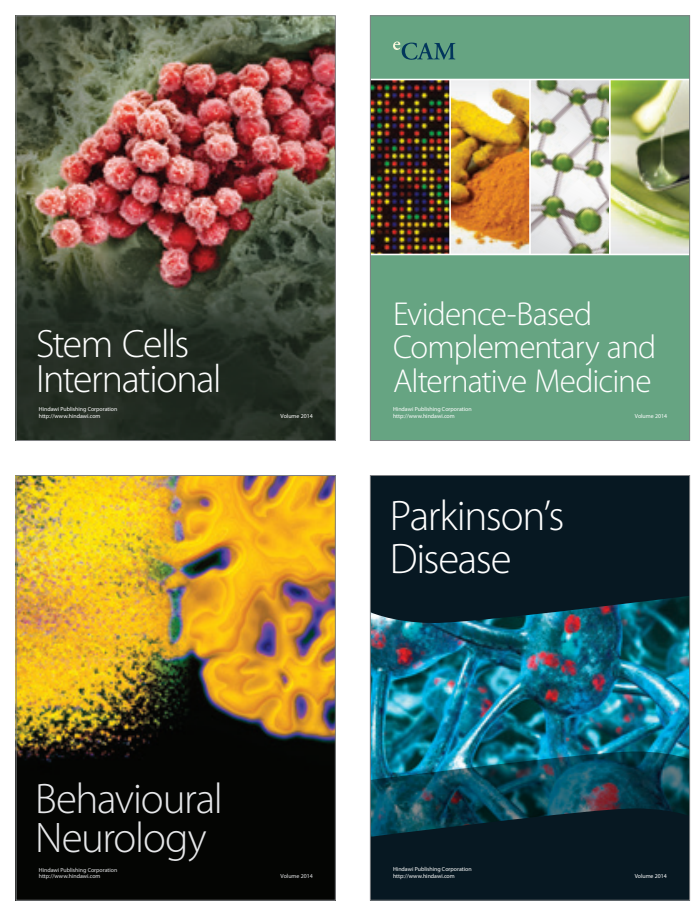

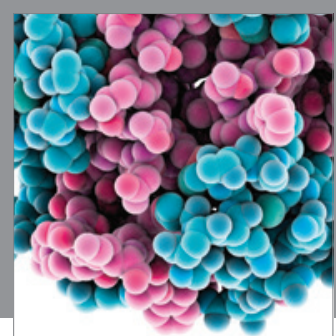

Journal of
Diabetes Research

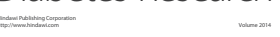

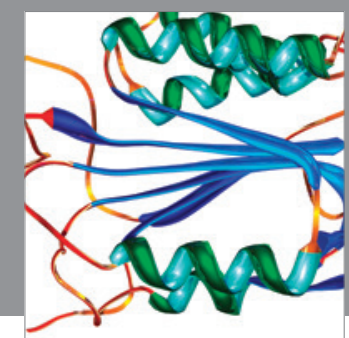

Disease Markers
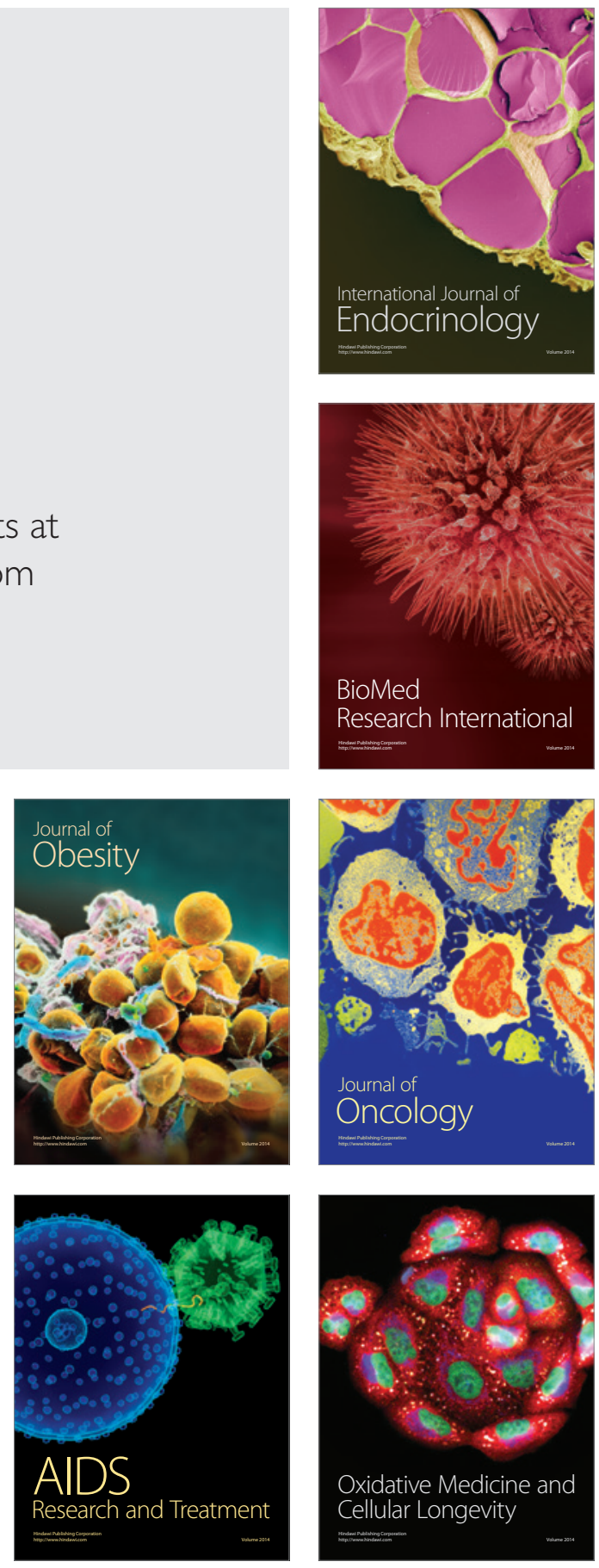